Summary/conclusions: This study provides proof-of-principle that ddPCR reliably detects fetal HPA-1a signals from early gestation, allowing clinicians time to assess the need for IVIg management. This assay has the potential to provide a safer approach for managing such alloimmunised antenatal cases.

\section{LIQUID BIOPSY FOR MANAGEMENT OF HAEMOLYTIC DISEASE OF THE FETUS AND NEWBORN}

Robert Flower $^{1}$, Helen O’Brien ${ }^{1}$, Glenda Millard ${ }^{1}$, Catherine Hyland $^{1}$, Glenn Gardener ${ }^{2}$

${ }^{1}$ Research and Development, Australian Red Cross Lifeblood, Kelvin Grove, Qld, Australia; and ${ }^{2}$ Mater Health Services, Raymond Terrace, South Brisbane, Qld, Australia

Background: Haemolytic disease of the fetus and newborn arises from maternal allo-antibodies to red cell antigens, crossing the placenta. The most frequent antibodies after $\mathrm{RhD}$, are Kell, Duffy and RhCE antigens.

Liquid biopsy in pregnancy management is concerned with the detection of cell-free fetal DNA (cffDNA) circulating in the maternal plasma. The accuracy for fetal $R H D$ genotyping is well established.

Aim: To evaluate a maternal blood test to predict the fetal, non$\mathrm{RhD}$, blood group status.

Methods: A clinical collaborative study assessed the accuracy for a suite of assays to predict fetal Kell, Duffy, Rhc/C and Rhe/E status for women with respective antibodies $(n=90$, gestation age 8-37 weeks).

Droplet digital PCR technology, ddPCR, tested for single nucleotide variants (SNVs) associated with these blood group antigens. Infant cord blood serotypes were used as the primary outcome measure.

Results: Fetal specific signals were detected as early as 8 weeks gestation. Genotyping matched all available infant cord outcomes 45/45. Accuracy is $100 \%$ (95\% confidence interval 91.96-100\%). Discussion: A clinical demand exists for liquid biopsy of maternal blood for fetal blood group genotyping. Droplet digital PCR is accurate and provides an evidence base to guide clinical care for at-risk pregnancies.

\section{UTILISING GENEXPERT TO IDENTIFY CML IN THE PRIMARY CARE SETTING: TRIGGERS FOR TESTING AND PREDICTORS OF POSITIVE RESULTS}

Elise Flynn, Ellen Maxwell

Department of Haematology, Melbourne Pathology, Collingwood, Vic, Australia

The GeneXpert utilises quantitative PCR to detect BCR-ABL fusion transcripts in the molecular monitoring of patient with chronic myeloid leukaemia (CML). ${ }^{1}$ Although GeneXpert hasn't currently been validated for the diagnosis of CML, BCR-ABL $\mathrm{PCR}$ on peripheral blood is frequently requested for this purpose. We performed a retrospective audit of BCR-ABL results in patients without established CML to assess test indications, triggers for ordering and the pre-test predictors of a positive BCR-ABL result. From January to November 2019, there were 178 tests with ten positive cases identified. All positive cases were initiated by a general practitioner triggered by a previous abnormal full blood count and film and had leucocytosis, neutrophilia and myeloid left shift, with nine cases having a basophilia. The presence of basophilia and myeloid left shift amongst the 178 patients was highly predictive of an underlying myeloid neoplasm or clonal myeloid mutation on subsequent molecular testing (PPV 100\% and 71\%, respectively). This reinforces our laboratory protocol of contacting general practitioners when these features are identified on blood film to expedite further testing and referral. In order to reduce unnecessary ordering of $\mathrm{BCR}-\mathrm{ABL}$, education is required regarding the low yield of this test in the absence of leucocytosis or neutrophilia.

\section{Reference}

1. Enjeti A, Granter N, Ashraf A, et al. A longitudinal evaluation of performance of automated BCR-ABL1 quantitation using cartridge-based detection system. Pathology 2015; 47: 570-4.

\section{METASTATIC GERM CELL TUMOUR PRESENTING AS ACUTE MEGAKARYOBLASTIC LEUKAEMIA AND DISSEMINATED MELANOMA: A CASE REPORT}

${\text { Hannah } \mathrm{Hsu}^{1}}^{1}$, Veena Gullapalli ${ }^{1,2}$

${ }^{T}$ Department of Haematology, Wollongong Hospital, Wollongong, Australia; and ${ }^{2}$ University of New South Wales, NSW, Australia

Since first recognised in 1985, studies have described the unique and rare association between haematological malignancies and germ cell tumours, most commonly acute megakaryoblastic leukemia (AMKL) and non-seminomatous germ cell tumours. A recent molecular study identified mutations in PTEN and TP53 in both tumours, supporting the theory that distinct genomic alterations underlie this rare association. It is also recognised that teratomatous components of mediastinal germ cell tumours have a tendency for secondary transformation to other malignancies including melanoma. We report a case of a 38-year-old man presenting with severe back pain. An MRI spine demonstrated diffuse marrow replacement and bony lesions concerning for metastatic disease. The patient had recently completed chemotherapy for a mediastinal germ cell tumour with teratomatous components on histopathology. A staging CT identified liver and splenic lesions suspicious for metastatic deposits. A peripheral blood film performed on admission was found to have circulating blasts with an immunophenotype consistent with megakaryoblasts. He subsequently underwent a bone marrow biopsy confirming AMKL with a TP53 mutation. A liver biopsy revealed necrotic tumour with immunohistochemistry consistent with metastatic melanoma. In summary, we describe a case of treated mediastinal germ cell tumour with secondary transformation to metastatic melanoma with synchronous AMKL.

\section{UNKNOWN UNKNOWNS: LIMITATIONS IN DETECTING TYPE II ANTITHROMBIN DEFICIENCY BY THE XA- BASED CHROMOGENIC METHOD}

\author{
Nathan Klose $^{1,2}$, Robyn Coleman ${ }^{1}$, Yasmin Harvey ${ }^{1}$, \\ Rebecca Adams ${ }^{1,2}$ \\ ${ }^{1}$ Sullivan Nicolaides Pathology, Bowen Hills, Qld, Australia; \\ and ${ }^{2}$ School of Medicine, University of Queensland, St Lucia, \\ Qld, Australia
}

Inherited deficiency of antithrombin (AT) occurs as a heterozygous quantitative reduction (type I) or qualitative reduction (type 